\title{
POLÍTICA DE PRECIOS DE GARANTÍA CONTRA APOYOS DIRECTOS: ANÁLISIS DEL BIENESTAR DEL PRODUCTOR
}

\section{SUPPORT PRICE POLICY VERSUS DIRECT PAYMENTS TO PRODUCERS: ANALISYS OF PRODUCERS' WELFARE}

\author{
Eligio Jiménez García ${ }^{1}$, Miguel A. Martínez Damián ${ }^{* 1}$ y Antonio Kido Cruz ${ }^{2}$
}

\begin{abstract}
${ }^{1}$ Programa de Economía, Colegio de Postgraduados-Campus Montecillo. Km. 36.5. Carretera México-Texcoco. 56230, Montecillo, Texcoco. Estado de México. Fax: 01(595) 952-0281. ${ }^{2}$ Instituto de Investigaciones Económicas y Empresariales (ININEE), Ciudad Universitaria, Universidad Michoacana de San Nicolás de Hidalgo. Morelia, Michoacán.
\end{abstract}

\section{RESUMEN}

Hasta el año 1993 el gobierno Mexicano intervino en el mercado de granos básicos mediante precios de garantía. En 1994 el gobierno crea el Programa de Apoyos al Campo (PROCAMPO) que aplica apoyos directos al ingreso monetario de los productores y evita la distorsión en precios inherente a otros esquemas de subsidio o apoyos a la producción. En esta investigación se analizó el efecto de estos dos tipos de política sobre el bienestar del productor a través de la medición y la comparación del excedente del productor, definido como la cantidad de dinero que le da a ganar al productor una política económica por encima de sus costos de producción. Se encontró que aunque el cambio de política económica produjo una reducción del excedente del productor agrícola por la caída de precios de granos básicos, los apoyos otorgados por el PROCAMPO compensaron la pérdida de ingreso monetario. Al considerar una canasta de cultivos básicos que incluía maíz, frijol, trigo, arroz y sorgo, y suponer que el excedente del productor es una buena medida del bienestar del productor, el bienestar del productor no se redujo durante el periodo 1986-2004, debido a que los apoyos directos del PROCAMPO sobre-compensaron en tres veces la pérdida de ingreso causado por la reducción de precios.

Palabras clave: Procampo, excedente del productor, canasta básica.

\section{SUMMARY}

Until 1993 the Mexican government implemented a farm policy based on governmental intervention in the basic crops market through a support price program. In 1994 the PROCAMPO (Farm's Support Program) was created with the objective of applying direct payments to producers' income. In this study we analysed the economic effects of these two programs on the producers' welfare by means of measuring and comparing the "producer surplus", defined as the amount of money that producers earn over production costs under either economic policy. The analysis showed quantitative evidence that although the political economic change caused a reduction in the producer surplus due to the fall of cereal prices, the direct payments provided by PROCAMPO compensated that income loss. Considering a basket of basic crops which included corn, dry beans, wheat, rice and sorghum, and assuming that the producer surplus is a good measure of producer' welfare, this study conclude that the producers' welfare was not reduced during the period 1986-2004, because the direct payments provided by PROCAMPO over compensated by three times the income loss caused by the price reduction.

Index words: Procampo, producers' surplus, basic basket.

\section{INTRODUCCIÓN}

Durante cinco décadas, hasta 1993, el gobierno mexicano utilizó esquemas de política agrícola basados en la intervención gubernamental en el mercado de productos básicos a través de precios de garantía, subsidios a los insumos y apoyos a la comercialización, para garantizar un nivel de ingreso a los productores, mejorar los niveles de producción y estabilizar los precios de productos agrícolas (SARH, 1994).

En 1994 se creó el programa PROCAMPO con el objetivo de dar apoyos directos a los productores y evitar distorsiones en precios inherentes a cualquier esquema de subsidio o apoyo a la producción; se buscaba así atacar la pobreza al distribuir el gasto público agropecuario de manera equitativa, sin perder de vista el mejor uso de los recursos de la economía (Téllez, 1994). Investigadores como Rubio y Vélez (1994) previeron impactos en la estructura productiva y en el padrón de cultivos del país y advirtieron que muchos productores de granos básicos dejarían esta actividad para dedicarse al cultivo de frutas y hortalizas por la desventaja en competitividad respecto a los Estados Unidos de América y Canadá.

Posterior al cambio de tal política, de precios de garantía a pagos directos, se han desarrollado estudios para 
cuantificar sus efectos. Puente-González (2001) analizó cuantitativamente el cambio de política agrícola al comparar los presupuestos financieros y económicos de dos años (1991 y 1996), y concluyó que si se considera un patrón de cultivos más grande el efecto del cambio de política sería positivo en aspectos como rentabilidad, ganancia neta por hectárea y ventajas comparativas de la agricultura mexicana.

Aunque el cambio de política económica hacia pagos directos produjo una caída en precios reales, al liberar el mercado de productos agrícolas con el consecuente decremento en el ingreso de los productores, debe analizarse sí el apoyo del PROCAMPO compensa esa pérdida de bienestar.

Con la finalidad de aportar elementos cuantitativos al análisis del cambio de política de precios de garantía a pagos directos, aquí se evaluó el efecto sobre el bienestar del productor medido por el excedente del productor, definido éste como la cantidad de dinero que le da a ganar al productor una política económica por encima de sus costos de producción. Se aclara, sin embargo, que este análisis comparativo no considera el efecto directo de importaciones ni de posibles transferencias del excedente del productor al consumidor.

El objeto de medir y comparar el excedente del productor es conocer en qué sentido y magnitud cambió el ingreso de los productores, y determinar si el apoyo económico del PROCAMPO compensa la pérdida de ingreso ocasionada por la caída en precios al eliminar los precios de garantía. Se formula la hipótesis de trabajo de que el bienestar en términos de ingreso del productor no se reduce al cambiar la política económica de Precios de Garantía a la de Apoyos Directos al Productor.

\section{FUENTES DE INFORMACIÓN Y MÉTODOS}

La canasta de productos básicos que se estudia aquí está compuesta por maíz (Zea mays L.), frijol (Phaseolus vulgaris L.), trigo (Triticum vulgare L.), arroz (Oriza sativa L.) y sorgo (Sorghum bicolor L. Moench), debido a que son cultivos que participan tanto en el programa precios de garantía como en el de apoyos directos, condición necesaria para hacer la comparación de excedentes del productor. Además, en México estos cultivos son importantes en superficie cultivable y desde el punto de vista económico. Se analiza el periodo de 1986 a 2004, del cual ocho años corresponden a la política de Precios de Garantía (1986-1993) y 11 años a la política de Apoyos Directos al Productor (1994-2004).
Se utiliza información anual de precios al productor, cantidades producidas de los productos estudiados y montos de apoyo otorgados a los productores, proveniente del Servicio de Información y Estadística Agroalimentaria y Pesquera (SIAP) de la Secretaría de Agricultura, Ganadería, Desarrollo Rural y Pesca (SAGARPA) ${ }^{1}$, la Organización para la Cooperación Económica y el Desarrollo $(\mathrm{OECD})^{2}$ y el Programa de Apoyos al Campo (Procampo $)^{3}$. Se utiliza el Índice Nacional de Precios al Productor (INPP) agregado, base $1994=100$, del Banco de México para deflactar los precios.

Se asumen como ciertos los siguientes supuestos: 1) Las Unidades de Producción Agrícola son empresas que producen bienes, el propietario toma decisiones de qué, cómo y cuánto producir, y obtiene beneficios o pérdidas de tales decisiones; 2) El productor actúa racionalmente para maximizar el beneficio que obtiene de la producción y venta de sus bienes; y 3) Se cumple la ley de la oferta que establece que la oferta del bien reacciona de manera positiva ante cambios en el precio.

Las elasticidades precio de la oferta $\left(E_{0}\right)$ de los cultivos, utilizadas para calcular los excedentes del productor, se obtienen de modelos de oferta nerlovianos con base en el precio por tonelada de producto $(p)$. Para el caso del maíz se introduce como variable explicativa a las transferencias al productor estimadas $\left(\mathrm{TPE}^{4}\right)$, para calcular mejor la respuesta del productor ante el estímulo económico en circunstancias de fuerte intervención gubernamental, cuantificado mediante la metodología y la información estadística de la Organización para la Cooperación y el Desarrollo Económico (OECD, 2005) según el manual: "Methodology for the measurement of support and use in policy evaluation" .

Para obtener los modelos de respuesta de la oferta de cada producto se plantea la siguiente función:

$$
q_{t}^{d}=\alpha_{1}+\alpha_{2} p_{t}^{e}+\alpha_{3} z_{t}+u_{t}
$$

Donde la cantidad a producir deseada en el periodo $t$ es una función de los precios relativos esperados y de un

\footnotetext{
${ }^{1}$ Información consultada de la página www.siap.sagarpa.gob.mx (julio 2006).

${ }^{2}$ Información consultada de la página www.oecd.org (agosto 2006).

${ }^{3}$ Información consultada de la página www.aserca.gob.mx (julio 2006).

${ }^{4}$ Se define al TPE (transferencias al productor estimadas) como un indicador del valor monetario anual de las transferencias brutas de los consumidores y contribuyentes a los productores agrícolas medido a nivel de parcela, a través de la aplicación de instrumentos de política. (OECD, 2005).

${ }^{5}$ OECD. (2005). Methodology for the measurement of support and use in policy evaluation. Manual consultado en sitio web: www.oecd.org/dataoecd (agosto 2006)
} 
número de desplazadores de la oferta; $q_{t}^{d}$ es la cantidad producida deseada en el periodo $t$, en toneladas; $p_{t}^{e}$ es el precio esperado (un vector de precios), en pesos por tonelada; $z_{t}$ es un vector de desplazadores exógenos; y $u_{t}$ representa las perturbaciones estocásticas que afectan la producción, con valor esperado de cero. Los valores de $\alpha_{i}$ son los parámetros del modelo, con $\alpha_{2}$ igual al coeficiente del precio de la función de oferta (Sadoulet y Janvry, 1995).

Al considerar el ajuste parcial de las cantidades producidas y la formación de expectativas en precios, se obtiene la función de oferta en forma reducida:

$q_{\mathrm{t}}=\pi_{1}+\pi_{2} p_{\mathrm{t}-1}+\pi_{3} q_{\mathrm{t}-1}+\pi_{4} q_{\mathrm{t}-2}+\pi_{5} z_{t}+\pi_{6} z_{\mathrm{t}-1}+e_{t}$

La respuesta de la oferta respecto al precio de corto plazo está dada por $\pi_{2}$, y de largo plazo por $\alpha_{2}=\pi_{2} / \delta \gamma \geq \pi_{2}$; con $\delta$ igual al coeficiente de ajuste parcial de la cantidad, y $\gamma$ el respectivo coeficiente de ajuste parcial en precio (Sadoulet y Janvry, 1995).

Para los cultivos de trigo y arroz se utilizó el modelo nerloviano restringido que no incluye desplazadores de la oferta dado que el modelo explica adecuadamente el comportamiento de la variable dependiente a un nivel de significancia de $5 \%$, en este caso $\alpha_{2}=-\pi_{2} /\left(\pi_{3}+\pi_{4}-1\right)$.

Los parámetros de las funciones de oferta $\left(\pi_{i}\right)$ se estimaron con máxima verosimilitud al considerar la corrección por autocorrelación en los términos del error. Se utilizó el programa estadístico SAS con el procedimiento PROC MODEL, que hace una estimación de los parámetros bajo la forma no lineal.

El cálculo del excedente del productor (EP) se hizo mediante la cuantificación de las áreas (1 y 2) por encima de la curva de oferta y debajo del precio. Se analizó solamente el efecto del precio sobre la cantidad ofrecida del año $t$ a $t+1$. (Figura 1). Es decir, se hizo el cálculo del área 1 y 2 para años contiguos.

El cambio del excedente del productor $(\triangle E P)$ ante un decremento en el precio está dado por:

$$
\Delta E P=q^{0}\left(p^{1}-p^{0}\right)-G S N P
$$

Donde la ganancia social neta en producción (GSNP) ante una disminución en el precio, igual al área 2 (Figura 1), se mide por:

$$
G S N P=\frac{1}{2} E_{0} t^{2} W=\frac{\left(q^{0}-q^{1}\right)\left(p^{0}-p^{1}\right)}{2}
$$

Donde: $p^{0}=$ precio doméstico del bien producido en $t ; p^{l}$ $=$ precio doméstico del bien producido en $t+1 ; q^{0}=$ cantidad producida al precio inicial; $q^{l}=$ cantidad producida después del cambio en precios; $E_{0}=$ elasticidad arco de la oferta:

$\left[\frac{q^{1}-q^{0}}{p^{1}-p^{0}}\right]\left[\frac{p^{0}}{q^{0}}\right] ; t=\left\lfloor\left(p^{1}-p^{0}\right) / p^{0}\right\rfloor=\left(p^{1} / p^{0}\right)-1$

= tasa de cambio en precios; y $W=p^{0} q^{0}=$ valor de la producción en las condiciones iniciales.

Precio

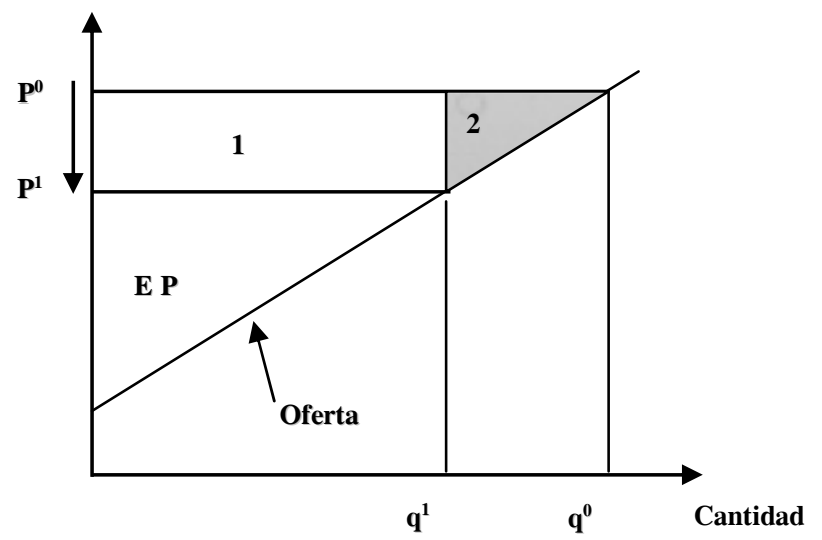

Figura 1. Efecto del decremento del precio de un bien sobre el bienestar del productor. Abreviaturas: $p^{0}$ es el precio al año inicial $t ; p^{1}$ es el precio al año siguiente $t+1 ; q^{0}$ es la cantidad producida al año inicial $t ; q^{1}$ es la cantidad producida al año siguiente $t+1 ; 1$ y 2 corresponden a la pérdida de excedente del productor (EP).

Con las expresiones anteriores se calcularon los cambios en el excedente del productor (EP) de un año a otro para cada cultivo y para el periodo de estudio (19862004). Posteriormente se comparó el cambio del EP con las aportaciones realizadas por el PROCAMPO para el periodo 1994-2004, para conocer en qué medida se compensa la pérdida de ingreso monetario de los productores de granos básicos.

\section{RESULTADOS Y DISCUSIÓN}

Los modelos nerlovianos de respuesta de la oferta con sus resultados estadísticos (Cuadro1) indican que en el caso del maíz el modelo explicó $87 \%$ de variación de la cantidad producida $(q)$ y el nivel de significancia más bajo 
$(p \text {-value })^{6}$ al que puede rechazarse la hipótesis nula de que el precio $\left(p_{t-1}\right)$ no explica el comportamiento de $q=0$, fue de 0.39; en tanto, el modelo de frijol explicó sólo 25 $\%$ de la variación en la cantidad producida $(q)$, y su valor $p$-value del parámetro del precio fue de 0.46. Este resultado contrastante para frijol indica la poca respuesta a precios que despliega la propia función oferta.

El modelo correspondiente a trigo explicó $73 \%$ del comportamiento de la cantidad producida $(q)$ y el $p$-value para el parámetro del precio $\left(p_{t-1}\right)$ fue de 0.031 . El modelo del arroz explicó $56 \%$ del comportamiento de la cantidad producida $(q)$, y el $p$-value para el parámetro del precio $\left(p_{t-1}\right)$ fue de 0.052. Por último, el modelo del sorgo explicó $56 \%$ de la cantidad producida $(q)$ y el $p$-value para el parámetro del precio $\left(p_{t-1}\right)$ fue de 0.136 .

Las estadísticas de prueba son aceptables para trigo, arroz y sorgo; no así para maíz y frijol. Para efectos de estimación del cambio en el excedente del productor de la canasta maíz, frijol, trigo, arroz, sorgo se utilizaron los resultados de los cinco cultivos básicos, tal como se obtuvieron. Para maíz y frijol la falta de ajuste y la menor significancia indica que es posible que exista otra forma de modelar su oferta con mayor precisión; sin embargo, aquí se trató conjuntamente a los cinco cultivos bajo una especificación nerloviana de la oferta.

En la Figura 2 se observa la tendencia decreciente seguida por los precios de los granos básicos en estudio durante el periodo 1986-2004. Si bien la tendencia decreciente es para todo el periodo de estudio, se enfatiza que los precios más reducidos ocurrieron en los años posteriores a 1994. Por ejemplo, la diferencia porcentual en nivel promedio de precios para el periodo de apoyos directos comparado con el periodo de precios de garantía, fue de: $-32.1,-10.8,-20.1,-24.1$ y $-23.0 \%$, respectivamente para maíz, frijol, trigo, arroz y sorgo.

Los valores de respuesta de la oferta de corto plazo y la elasticidad oferta estimada para el cálculo del cambio en el excedente del productor (Cuadro 2), indican que para los cinco cultivos la elasticidad es menor a la unidad, lo que implica poca respuesta de la cantidad ofrecida a cambios en precio. Esto se explica por la naturaleza de esos cultivos asociados con actividades de producción primaria. Se utilizó el parámetro de respuesta de la oferta

\footnotetext{
${ }^{6}$ El valor $p$ o " $p$-value", se conoce como el nivel observado o exacto de significancia o la probabilidad exacta de cometer un error tipo 1. Técnicamente, el valor $p$ está definido como el nivel de significancia más bajo al cual puede rechazarse una hipótesis nula.
}

de corto plazo dado que el cálculo del excedente del productor se hizo en dos años consecutivos.

Los resultados de cambio del excedente del productor, los montos aportados por el PROCAMPO en cada periodo, así como la suma de los montos anteriores y el porcentaje cubierto por el PROCAMPO (Cuadro 3), muestran que durante el periodo 1986-2004 el excedente del productor se redujo debido a cambios en el precio de los productos de la canasta M-F-T-A-S. Solamente los productores de maíz del periodo 1986-1993 obtuvieron un incremento de su excedente debido al precio, aunque tuvieron un decremento en el periodo completo.

Con excepción del producto arroz, durante el periodo 1994-2004 las pérdidas en el excedente del productor se incrementaron debido a la caída de precios reales derivado de la liberalización del mercado de granos básicos. Durante este periodo se brindaron apoyos directos al productor a través del PROCAMPO para compensar las pérdidas ocasionadas por la disminución de precios. Para este periodo, el PROCAMPO representó 7.95 veces la pérdida de excedente del productor en frijol y 4.91 veces la pérdida en sorgo. Esta proporción para los demás granos (maíz, trigo y arroz) se estimó respectivamente en 2.87, 2.07 y 2.15 .

Al considerar todo el periodo de estudio (1986-2004), los resultados indican que el apoyo del PROCAMPO compensó la pérdida de excedente del productor atribuible a la caída de precios; en el caso del frijol, éste representó 10.95 veces la pérdida de ingreso.

Bajo las consideraciones planteadas en este estudio y al analizar la canasta de granos básicos (M-F-T-A-S) en su conjunto, se estima una pérdida de 733 millones de pesos del excedente del productor debido al factor precio para el periodo 1986-1993, y de 10753 millones para el periodo 1994-2004, que en suma representaron una pérdida de 11486 millones de pesos para el periodo 1986-2004 (Figura 3).

Las aportaciones directas realizadas por el PROCAMPO para los cinco granos básicos sumaron 36860 millones de pesos, que corresponden a 3.2 veces la pérdida atribuible a la caída de los precios por el cambio de política agrícola. El balance arrojó un incremento de 25373 millones de pesos en el ingreso neto del productor agregado para el periodo 1986-2004. 
Cuadro 1. Coeficientes de respuesta de la cantidad ofertada de algunos cultivos básicos. México, 1986-2004.

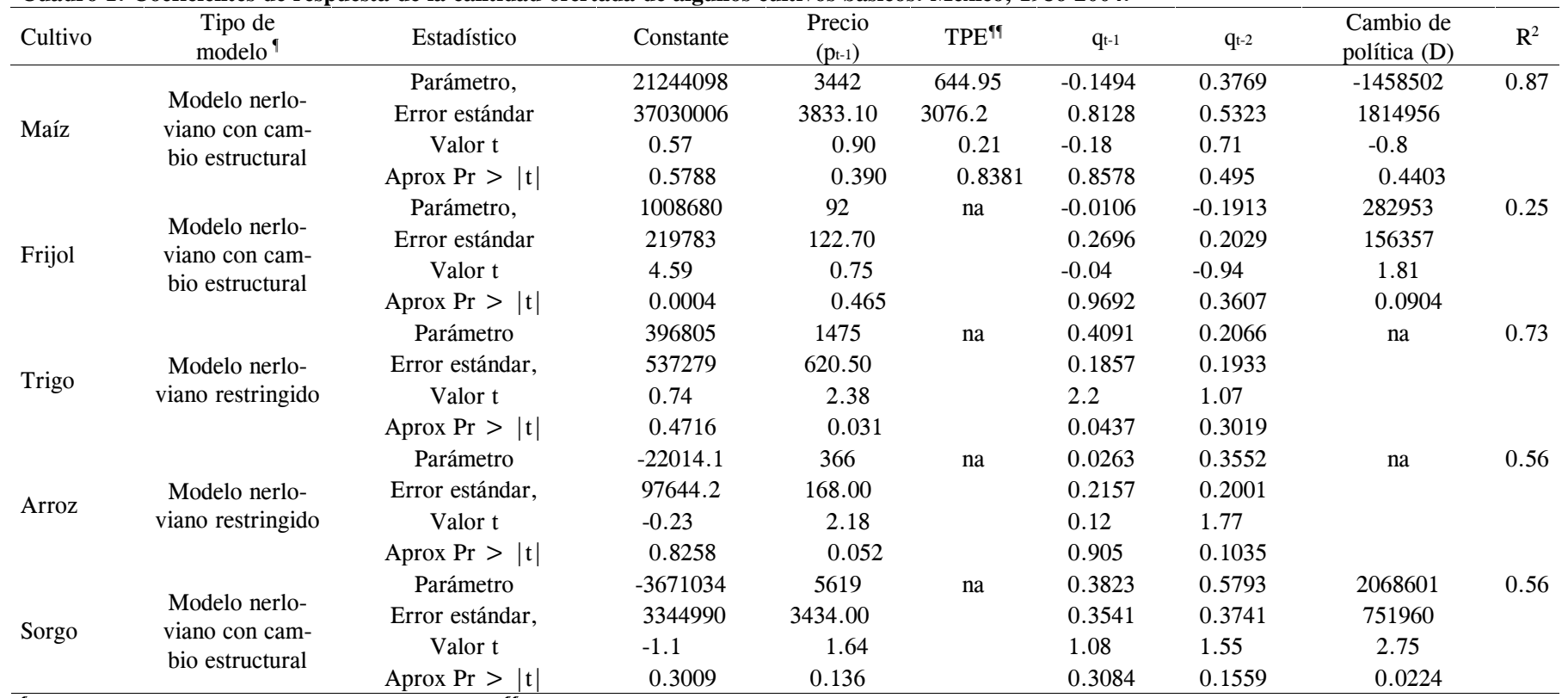

' Con ajuste parcial y formación de expectativas. "' TPE = Transferencia al productor estimada; na = Variable no utilizada.

Fuente: Elaboración propia con base en datos de SIAP-SAGAQPA, OECD y PROCAMPO.

Cuadro 2. Respuesta de la oferta de cultivos básicos. México 1986-2004.

Respuesta de la oferta de corto plazo

\begin{tabular}{lc}
\cline { 2 - 2 } Cultivo & Precio $\left(\mathrm{p}_{\mathrm{t}-1)}\right.$ \\
\hline Maíz & 3442 \\
Frijol & 92 \\
Trigo & 1475 \\
Arroz & 366 \\
Sorgo & 5619
\end{tabular}

Elasticidad de la oferta de corto plazo

\begin{tabular}{c}
\hline Precio $\left(\mathrm{p}_{\mathrm{t}-1}\right)$ \\
\hline 0.14 \\
0.17 \\
0.24 \\
0.60 \\
0.47
\end{tabular}

Fuente: Elaboración propia con base en datos de SIAP-SAGAQPA, OECD y PROCAMPO.

Cuadro 3. Cambio en el excedente del productor debido al precio y apoyos del PROCAMPO. México, 1986-2004. Montos en pesos de 1994.

\begin{tabular}{|c|c|c|c|c|c|}
\hline Cultivos & Periodo & $\begin{array}{c}\Delta \mathrm{EP} \\
\text { (Mill. de pesos) }\end{array}$ & $\begin{array}{l}\text { PROCAMPO } \\
\text { (Mill. de pesos) }\end{array}$ & $\begin{array}{c}\Delta \mathrm{EP}+\mathrm{PROCAMPO} \\
\text { (Mill. de pesos) }\end{array}$ & PROCAMPO/AEP \\
\hline \multirow{3}{*}{ Maíz } & $1986-1993$ & -430.25 & 0.00 & -430.25 & 0.00 \\
\hline & 1994-2004 & -7640.13 & 21921.96 & 14281.23 & 2.87 \\
\hline & 1986-2004 & -8070.38 & 21921.96 & 13851.58 & 2.72 \\
\hline \multirow[t]{3}{*}{ Frijol } & 1986-1993 & 243.82 & 0.00 & 243.82 & 0.00 \\
\hline & 1994-2004 & -889.19 & 7069.01 & 6179.82 & 7.95 \\
\hline & 1986-2004 & -645.37 & 7069.01 & 6423.64 & 10.95 \\
\hline \multirow[t]{3}{*}{ Trigo } & 1986-1993 & -13.24 & 0.00 & -13.24 & 0.00 \\
\hline & 1994-2004 & -994.73 & 2057.01 & 1062.28 & 2.07 \\
\hline & 1986-2004 & -1007.97 & 2057.01 & 1049.04 & 2.04 \\
\hline \multirow[t]{3}{*}{ Arroz } & 1986-1993 & -317.87 & 0.00 & -317.87 & 0.00 \\
\hline & 1994-2004 & -81.06 & 173.93 & 92.87 & 2.15 \\
\hline & 1986-2004 & -398.93 & 173.93 & -225.00 & 0.44 \\
\hline \multirow[t]{3}{*}{ Sorgo } & $1986-1993$ & -215.61 & 0.00 & -215.61 & 0.00 \\
\hline & 1994-2004 & -1148.39 & 5638.25 & 4489.86 & 4.91 \\
\hline & 1986-2004 & -1363.99 & 5638.25 & 4274.26 & 4.13 \\
\hline
\end{tabular}

Fuente: Elaboración propia con base en datos de SIAP-SAGAQPA, OECD y PROCAMPO. 
MAÍZ

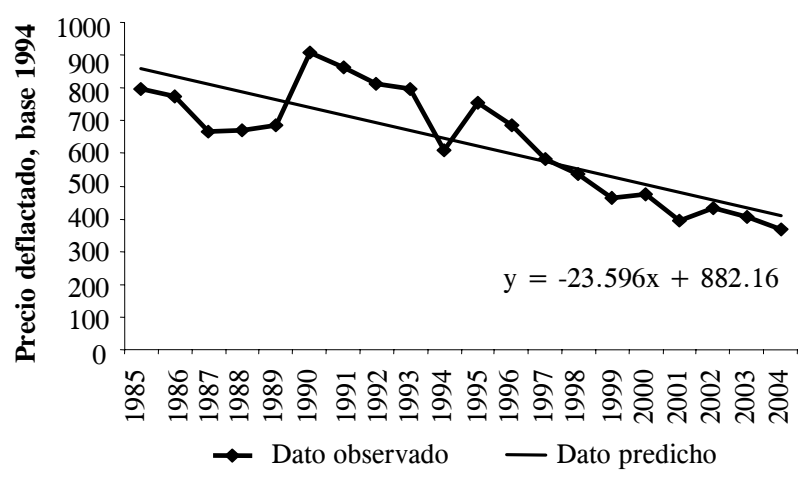

Año

TRIGO

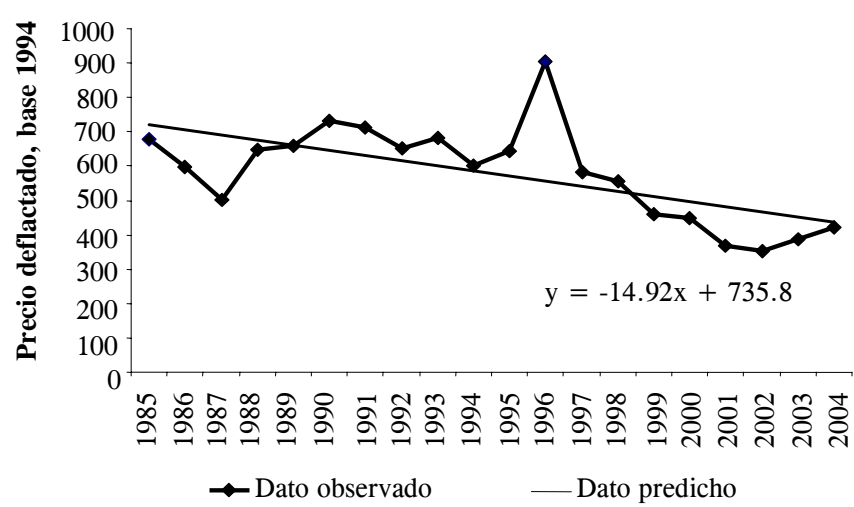

Año
FRIJOL

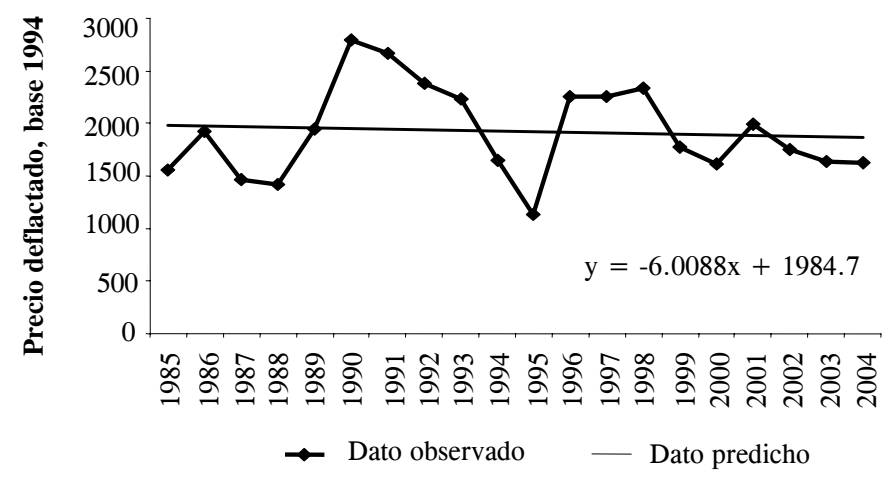

Año

ARROZ

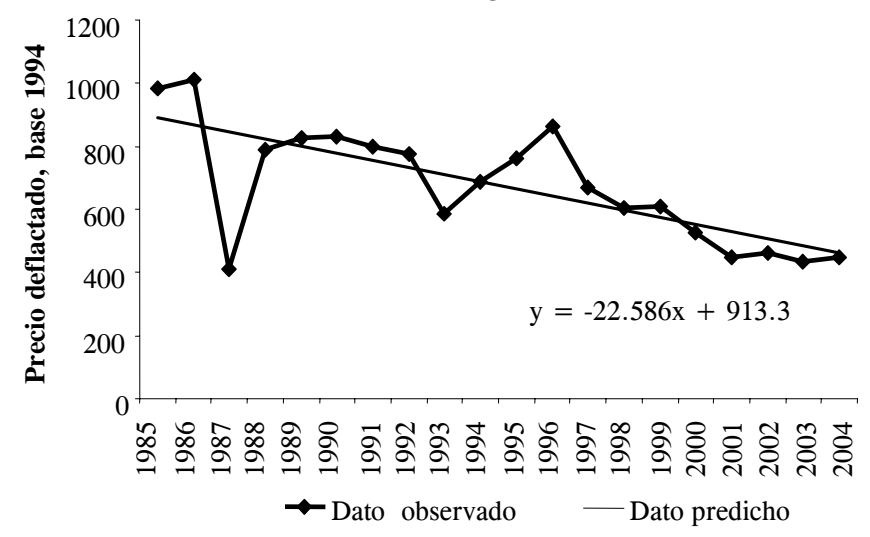

Año

SORGO

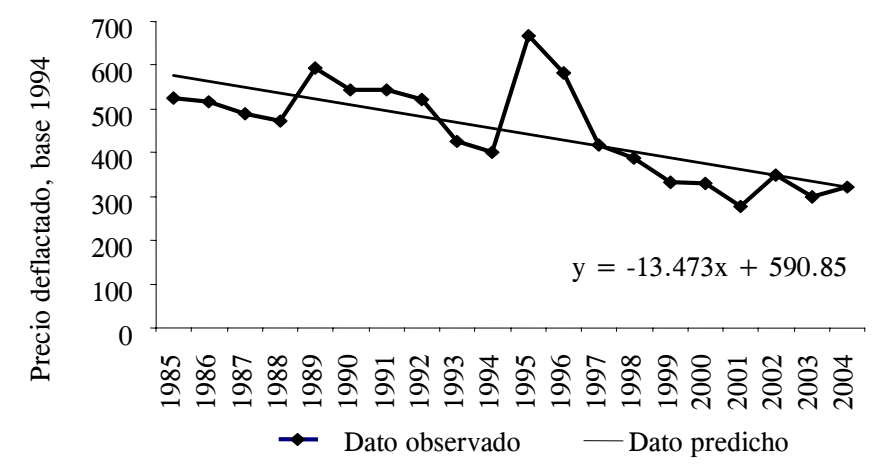

Año

Figura 2. Tendencias de precios reales (\$/t) de la canasta maíz, frijol, trigo, arroz y sorgo. México, 1986-2004. Fuente: Elaboración propia con base en datos de SIAP-SAGAQPA, OECD y PROCAMPO. 


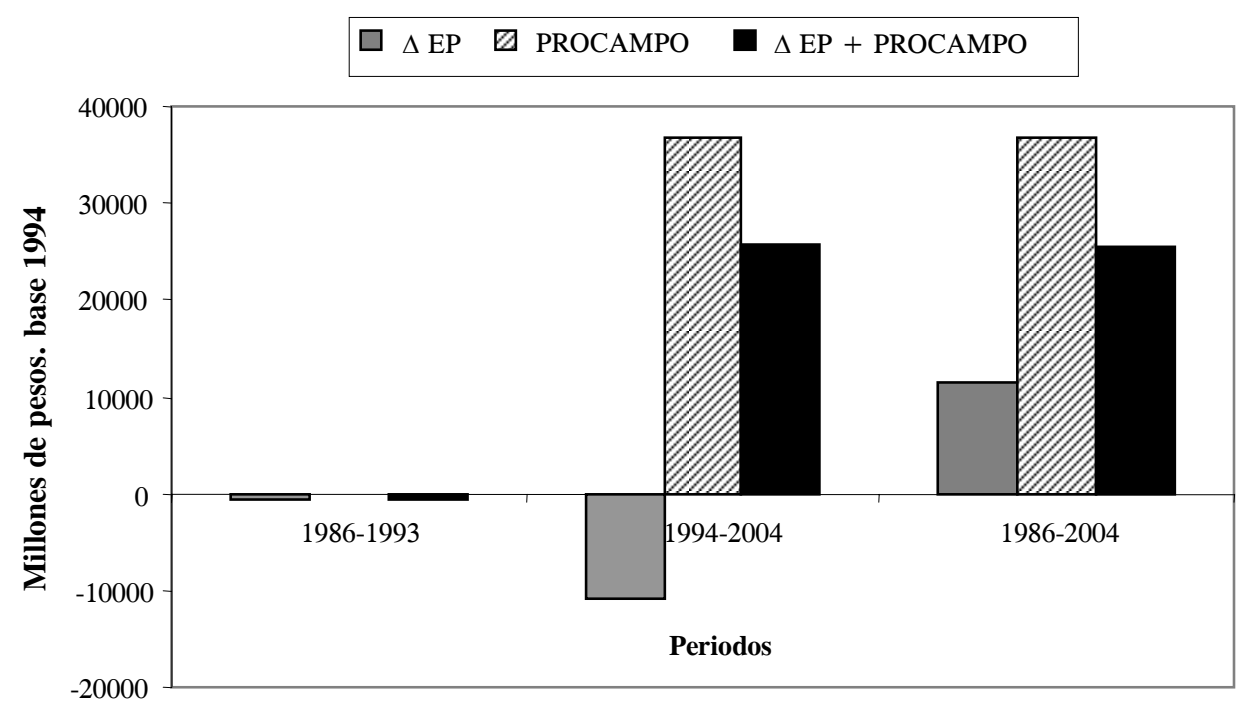

Figura 3. Cambios del excedente del productor y apoyos PROCAMPO para la canasta de granos básicos (maíz, frijol, trigo, arroz y sorgo). México, 1986-2004.

\section{CONCLUSIONES}

Sin considerar efectos de importaciones ni posibles trasferencias del productor al consumidor, este análisis presenta evidencia cuantitativa de que aunque el cambio de política económica produjo una reducción del excedente del productor agrícola por la liberalización del mercado de productos agrícolas, los apoyos otorgados por el PROCAMPO compensaron con suficiencia esa pérdida de ingreso.

Con excepción del arroz, durante el periodo 19942004 se incrementaron las pérdidas de excedente del productor debido a la caída de los precios reales derivado de la liberalización del mercado de granos básicos. Al considerar la canasta de granos básicos maíz-frijol-trigoarroz-sorgo y al suponer que el excedente del productor es una buena medida de su bienestar, se concluye que durante el periodo 1986-2004 el bienestar del productor en el agregado no se redujo, ya que los apoyos directos otor- gados por el PROCAMPO compensaron en tres veces el valor de las pérdidas causadas por la caída de precios.

\section{BIBLIOGRAFÍA}

Puente González A (2001) La Agricultura de México, Antes y Después de las Reformas Económicas de los Noventa. Un análisis nacional y regional en el Distrito de riego "Rio Yaqui". Center for Enviromental Science and Policy / Institute for International Studies. Stanford University. USA. 191 p.

Rubio G M, Vélez F (1994) El Impacto del TLC en el Campo Mexicano, el Impacto Sectorial del TLC en la Economía Mexicana, Lo Negociado del TLC. Kessel (comp). Mc Graw Hill. México. $334 \mathrm{p}$.

Sadoulet E, A De Janvry (1995) Quantitative Development Policy Analysis. The John Hopkins University Press, Baltimore. USA. $72 \mathrm{p}$.

Secretaría de Agricultura y Recursos Hidráulicos, SARH (1994) PROCAMPO: Vamos al Grano para Progresar. Secretaría de Agricultura y Recursos Hidráulicos, SARH. México. 59 p.

Téllez K L (1994) La Modernización del Sector Agropecuario y Forestal. Fondo de Cultura Económica, México. 307 p. 